\title{
Prevalence of Low Back Pain and its Impact on Quality of Life among Taif University Students
}

\author{
Ayman Abdelbaky Ahmad ${ }^{1}$, Turki Abdulmuin Althobaiti ${ }^{2}$, Mohammed Saleem Alsofiany ${ }^{2}$, Moayad Abed \\ Altowairqi $^{2}$, Omar Eid Aljuaid ${ }^{2}$, Muhammad Abdullah Althobaiti ${ }^{2}$, Faris Mohammed Alamri ${ }^{2}$ \\ ${ }^{1}$ Department of Family medicine and Community Medicine, College of Medicine Taif University, Saudi Arabia \\ ${ }^{2}$ Medical Students, College of Medicine, Taif University, Saudi Arabia \\ *Corresponding author: Omar Eid Aljuaid; o-e-g@outlook.com
}

Received 25 August 2021;

Accepted 10 September 2021;

Published 13 September 2021

\begin{abstract}
Background: Lower back pain (LBP) is associated with increased pain intensity, physical and psychological disability, and increased dependence on pain medications causing adverse effects on the day-to-day quality of life (QOL). The study aimed to assess the prevalence of LBP among Taif University students and its impact on quality of life. Methods: A pretested self-administered questionnaire was distributed randomly and electronically to all students who gave consent to participated. The questionnaire was be divided into three parts. The first part included demographic details; the second part assessed the prevalence of Low back pain and associated data that was recorded using the LBP section of Standardized Nordic Musculoskeletal Questionnaire (SNMA); the third part consisted of the RAND 36-Item Short-Form Health Survey (SF-36) for assessing the quality of life. Result: In our study majority of the participants were females (87.3\%), and 55.3\% belonged to the 20-25 years age group. The fixed orthodontic related history showed 59.8\% had undergone the treatment for 1 to 3 years and $31.6 \%$ of the participants reported that they had removed the fixed braces for more than five years. Among these participants, 89.5\% ( $\mathrm{n}=34)$ reported that the fascia (space) closed between the upper frontal teeth after Frenectomy, and 65.8\% $(n=25)$ agreed that spaced between the two upper front teeth still closed after removing the retainer. Conclusion: The lifetime prevalence, annual prevalence, and point prevalence of LBP were found to be $57.9 \%, 73.1 \%$, and $47.1 \%$, respectively. The point prevalence was statistically higher among female students than male students ( $\mathrm{p}=0.003$ ). There were statistically significant differences seen in lifetime and point prevalence of LBP between different colleges. A linear regression model showed that the annual prevalence of LBP had a significant impact on the students' quality of life.
\end{abstract}

Keywords: Lower Back Pain, Quality of Life, Compliance, Musculoskeletal.

\section{Introduction}

Low back pain (LBP) affects $85 \%$ of the world's population and the third most frequent reason for a physician office visit, and the fourth most common diagnosis associated with surgical procedures. Low back pain is a symptom of a variety of illnesses ${ }^{[1]}$. Depression is a common finding with LBP ${ }^{[2]}$. LBP is associated with increased pain intensity, physical and psychological disability, and increased dependence on pain medications ${ }^{[3,4]}$. Low back pain is the common cause of activity limitation, and the second most frequent reason for a physician office visit worldwide ${ }^{[5]}$. LBP is the leading of socioeconomic burden on individuals, families, communities, industry and government, Aside from prevalence and cost, the effect of LBP on the workplace is substantial. Approximately 149 million workdays per year are lost as a result of LBP ${ }^{[6-8]}$. Previous studies found a higher level of pain severity and/or disability to be related to higher costs and a lower healthrelated quality of life ${ }^{[9-11]}$. Impairments to sleep are commonly reported in individuals with $\mathrm{LBP}$, with a $65 \%$ decrease in sleep quality ${ }^{[12]}$.

A study conducted on three thousand high school students found $41 \%$ has low back pain and $65.0 \%$ were sleepdeprived concerning academic pressure ${ }^{[13]}$.

A prospective study among 684 university students found a oneyear incidence of low back pain to be $31 \%$ which had an impact on their quality of life, work, and socioeconomics ${ }^{[14]}$.

A Poland study conducted on 1321 universities students found $43.4 \%$ reported mild, and more than $20 \%$ reported severe low back pain. About $60 \%$ found difficulty with long sitting and the other $50 \%$ with long-standing. on other hand over $20 \%$ expressed limitation and $4.4 \%$ had to stop their physical activity due to low back pain ${ }^{[15]}$.

A study done in Saudi Arabia showed a high prevalence of musculoskeletal pain among medical students particularly in relation to those with a history of depression, trauma, or psychosomatic symptoms ${ }^{[16]}$. 
A Japanese Cohort study suggests exposure to stressful life events may be associated with low back pain which had an impact on daily life ${ }^{[17]}$.

A study on 1163 health sciences students in Saudi Arabia found the Lifetime prevalence of low back pain was $56.6 \%$, with $90.3 \%$ reported minimal disability ${ }^{[18]}$.

A study was made on 1800 medical French students with a mean age of 23 years old showed $68.9 \%$ prevalence of LBP which had a severe repercussion on the students work, quality of sleep, and their personal life ${ }^{[19]}$.

A study done on 640 medical students of Taif University reported $33.3 \%$ of low back pain, with the main risk factors were being a 2nd-year medical student, female gender, and high working hours and there was no significant association of psychological stress to low back pain ${ }^{[20]}$.

We didn't see a study interface low back pain with its impact on quality of life in university students of Saudi Arabia. We aimed to find the prevalence of low back pain and its impact on quality of life among students of Taif University in relation to deferent specialties

\section{Subjects and Methods}

Study design, time frame and setting: Our study was conducted at Taif city in KSA between 7st November 2020 to 30 March, 2021. A cross-sectional design was used Study population and Sampling specifications: a Sample size calculator website (Rasoft) was used with a margin of error of $5 \%$ confidence level of $95 \%$, population size of 42765 . The recommended sample size was 381 students across the university and our study was concluding 401 participants. The study included both male and female of Taif University students, who were 18 years of age and above and accepted to take part in the study the consent was taken electronically.

Tools of data collection: A self administered questionnaire was done, although Due to covid-19 pandemic the questionnaires distributed randomly and electronically either through E-mail or social media applications or both. The questionnaire was divided into 3 parts. The first part included demographic questions regarding age, sex, and name of specialty. The second part included Low back pain section of Standardized Nordic Musculoskeletal Questionnaire (SNMA) used to evaluate recent occurrence, pain experienced within previous year, and over lifetime, pain influence on Occupation, physical activity, pain causes hospital visit or conducted treatment, which has been proven it's reliability and validity ${ }^{[21]}$. Third part included questions to asses quality of life using the reliable and valid Arabic version of 36-Item Short Form Health Survey (SF-36), It consists of 36 items in 8 domains that assess the patient's physical and mental status ${ }^{[22]}$.

\section{Statistical analyses and sample size calculation:}

Statistical analysis and data management: All the data received from the survey were transferred into the MS Excel working sheet and then was analyzed using a Statistical Package for Social Sciences (SPSS) Ver.20. Descriptive statistics using frequencies and percentages were used to present categorical variables. The mean differences in scores for each domain in 36-Item Short Form Survey (SF-36) between concerned categorical variables were compared using Student's ' $t$ ' test and/or Analysis of Variance (ANOVA). Any possible association between categorical variables was analyzed using Pearson's Chi-square test. A significance value (p) less than 0.05 were considered statistically significant.

\section{Ethical considerations}

All participants written consents from their parents and willing to be interviewed and examined if they agree or not to take part in the study. Only those who agreed to participate were included. Before conducting any study-related procedures, ethical approval was obtained from Research Ethics Committee at Taif University, Saudi Arabia.

\section{Results}

Our study assessed the prevalence of low back pain and its impact on Taif University students' quality of life in different specialties. The prevalence of pain was evaluated using the Low back pain section of the Standardized Nordic Musculoskeletal Questionnaire (SNMA) and the health-related Quality of life among cardiovascular patients during the COVID-19 pandemic using the Arabic version of the RAND 36-Item Short Form Survey. The study included 401 participants who gave consent to participate that had $47.1 \%$ females, and $52.9 \%$ males from different institutions under Taif University. The sociodemographic characteristics of the participants are given in [Table 1].

\section{Lower back pain}

The lifetime prevalence of lower back pain as reported by the participants in this study was found to be $57.9 \%(\mathrm{n}=232)$, whereas the annual prevalence and point prevalence was found to be $73.1 \%$ $(n=293)$ and $47.1 \%(n=189)$. When the three types of prevalence were compared between two genders, it was observed that point prevalence of lower back pain was more reported in females (55\%) compared to males (40.1\%), which showed a statistically significant difference $(p=0.003)$. There were no statistically significant differences seen in annual prevalence and lifetime prevalence of lower back pain between the two genders. Similarly, there were no statistically significant differences observed in annual prevalence, point prevalence, and lifetime prevalence of lower back pain between participants Body mass index and also between different academic years [Table 2].

When we compared the lower back pain prevalence according to different colleges, it was found that the annual prevalence was comparatively more reported among students of College of Medicine (80.7\%), Administrative and Financial Sciences $(87.1 \%)$, and Engineering (83.3\%) compared to students from other colleges $(\mathrm{p}=0.007)$. There was no statistically significant difference seen in point prevalence of lower back pain between students from different colleges $(p=0.159)$. The lifetime prevalence of lower back was found to be in students from College of Applied Medical Sciences (73.2\%), Engineering (77.8\%), and Administrative and Financial Sciences (77.4\%) compared to other colleges, which showed statistically significant differences $(\mathrm{p}=0.003)$ [Table 3].

\section{Quality of life in participants with lower back pain:}

The impact of lower back pain on Quality of life among participants $(n=232)$ was assessed using the RAND 36-Item Health Survey. The 36-Item form is subdivided into nine subdomain scales, namely: Physical functioning, role limitations due to physical health, role limitations due to emotional problems, Energy/fatigue, Emotional wellbeing, Social functioning, Pain, General health, and overall health change, where a higher score showed better Quality of life. The internal consistency of each domain and its means are given in [Table 4].

When the scores of each domain were compared between two genders, it was found that the scores of the domain 'Energy/fatigue were significantly higher (better) in males (47.4 \pm 
23.3) compared to female students $(38.3 \pm 23.4), \mathrm{p}=0.004$. The scores for the domain 'pain' were reported higher in males $(71.8 \pm$ 25.3) compared to females $(65.4 \pm 23.9), \mathrm{p}=0.049$. The scores for general health were also better in males $(63.3 \pm 17.1)$ compared to females (58.8 \pm 17.2), $\mathrm{p}=0.046$ [Table 5].

The comparison of scores based on the participants' academic year showed statistically significant differences in all domains except for two domains (Role limitations due to emotional problems and Overall health change). The scores for Physical functioning were found to be higher in the sixth year $(71.4 \pm 32.8)$ and third-year students $(66.0 \pm 31)$ compared to others $(\mathrm{p}=0.007)$. The scores for Role limitations due to physical health were observed higher among Fifth $(76.8 \pm 33.3)$, sixth-year dental students $(76.9 \pm 36.4)$, and interns and graduates $(71.4 \pm 36.6)$ compared to others $(\mathrm{p}=0.010)$. The interns and graduates comparatively showed statistically significant higher scores for energy/fatigue (61.4 \pm 21.5$)$, emotional wellbeing (71.4 \pm 23 ), social functioning $(78.6 \pm 18.7)$, and General health $(78.6 \pm 16.5)$. The pain scores were comparatively higher among the sixth year $(81.8 \pm 18.9)$, Fourth year $(80.5 \pm 20.2)$, and fifth-year $(76.9 \pm$ 22.0), $\mathrm{p}<0.001$ [Table 6].

When we compared the scores of all the domains were compared between students of different colleges, the score for Physical functioning was found to be higher among students of College of Arts $(73.0 \pm 34.7)$, and lower scores were observed among College of Dentistry $(30.0 \pm 22.7)(\mathrm{p}=0.003)$. The scores for Role limitations due to emotional problems were found to be higher among students of College of Dentistry and lower among College of Design and Applied Arts ( $\mathrm{p}=0.043$ ). The Energy/fatigue scores were higher among College of Arts students, and lower scores were seen among students of College of Design and Applied Arts $(p=0.001)$. The emotional well-being scores were higher among College of Community Service and Continuing Education students and lesser among students of College of Design and Applied Arts $(\mathrm{p}<0.001)$. The social functioning scores were higher among College of Arts students and lesser among students of College of Dentistry $(\mathrm{p}=0.043)$. The pain scores were higher among College of Dentistry students and lowest among students of College of Design and Applied Arts ( $\mathrm{p}=0.012)$ [Table 7].

The comparison of scores of different domains based on the BMI of the students is given in Table 8. The scores were for Role limitations due to physical health was higher among students belonging to normal weight $(68.1 \pm 37.3)$, and lower scores were seen among underweight students $(\mathrm{p}<0.001)$. Students who were obese showed higher scores for Role limitations due to emotional problems $(p=0.014)$ and Emotional wellbeing $(p<0.001)$, whereas lower scores for both these domains were observed among underweight students. The general health scores were found to be comparatively higher in overweight students and lower among underweight students $(\mathrm{p}<0.043)$ [Table 8]

\section{Comparison of the Impact of LBP on QOL $(n=401)$}

To calculate the impact of lower back pain on the Quality of life, all the nine domains' scores were added. The mean scores for total Quality of life in students who had an annual prevalence of lower back pain were $512.6 \pm 172.5$, and in those who didn't have lower back pain was $593.7 \pm 149.9$, which showed a statistically significant difference $(\mathrm{p}<0.001)$ Similarly, there were statistically significant differences in QOL scores in students who had lifetime and point prevalence of lower back pain with those who didn't have any prevalence [Table 9]. A linear regression model was performed to see the levels of variability in the Quality of Life scores due to lower back pain by controlling the covariates: gender, academic year, College, and BMI. It showed a statistically significant difference in total Quality of life scores except for gender and College [Table 10]

Table 1: Sociodemographic characteristics of participants $(n=401)$

\begin{tabular}{|c|c|c|c|}
\hline & & Frequency & Percent \\
\hline \multirow[t]{2}{*}{ Gender } & Female & 189 & 47.1 \\
\hline & Male & 212 & 52.9 \\
\hline \multirow[t]{7}{*}{ Year } & First & 61 & 15.2 \\
\hline & Second & 59 & 14.7 \\
\hline & Third & 52 & 13.0 \\
\hline & Fourth & 75 & 18.7 \\
\hline & Fifth & 56 & 14.0 \\
\hline & Sixth & 91 & 22.7 \\
\hline & Intern or graduate & 7 & 1.7 \\
\hline \multirow[t]{13}{*}{ Collge } & Medicine & 145 & 36.2 \\
\hline & Applied Medical Sciences & 41 & 10.2 \\
\hline & Pharmacy & 27 & 6.7 \\
\hline & Dentistry & 4 & 1.0 \\
\hline & Community Service and Continuing Education. & 4 & 1.0 \\
\hline & Science & 21 & 5.2 \\
\hline & Engineering & 18 & 4.5 \\
\hline & Administrative and Financial Sciences & 31 & 7.7 \\
\hline & Computing and Information Technology & 38 & 9.5 \\
\hline & Arts & 15 & 3.7 \\
\hline & Design and Applied Arts & 15 & 3.7 \\
\hline & Faculty of Education & 23 & 5.7 \\
\hline & Sharia and Regulations & 19 & 4.7 \\
\hline \multirow[t]{4}{*}{ Body Mass Index } & Underweight & 67 & 16.7 \\
\hline & Normal weight & 176 & 43.9 \\
\hline & Overweight & 104 & 25.9 \\
\hline & Obesity & 54 & 13.5 \\
\hline
\end{tabular}


Table 2: Comparison of prevalence of prevalence of lower back pain with baseline characteristics

\begin{tabular}{|c|c|c|c|c|c|c|c|c|c|c|c|}
\hline & & & Anr & evalence & $\stackrel{\mathscr{g}}{\Xi}$ & & valence & $\cong$ & Life & back pain & $\cong$ \\
\hline & & & No & Yes & $\overrightarrow{2}$ & No & Yes & 2 & No & Yes & 2 \\
\hline & Female & $\mathrm{N}$ & 45 & 144 & & 85 & 104 & & 80 & 109 & \\
\hline$\frac{\overline{0}}{0}$ & & $\%$ & 23.8 & 76.2 & $\infty$ & 45.0 & 55.0 & & 42.3 & 57.7 & \\
\hline ల్ & Male & $\mathrm{N}$ & 63 & 149 & $\overrightarrow{0}$ & 127 & 85 & $\approx$ & 89 & 123 & 寸 \\
\hline & & $\%$ & 29.7 & 70.3 & & 59.9 & 40.1 & ๑ & 42.0 & 58.0 & 2 \\
\hline & Underweight & $\mathrm{N}$ & 13 & 54 & & 30 & 37 & & 28 & 39 & \\
\hline$\stackrel{\star}{x}$ & & $\%$ & 19.4 & 80.6 & & 44.8 & 55.2 & & 41.8 & 58.2 & \\
\hline$\underline{\Xi}$ & Normal weight & $\mathrm{N}$ & 55 & 117 & & 89 & 83 & & 71 & 101 & \\
\hline$\vec{y}$ & & $\%$ & 32.0 & 68.0 & ๖ & 51.7 & 48.3 & & 41.3 & 58.7 & \\
\hline$\sum^{\pi}$ & Overweight & $\mathrm{N}$ & 25 & 79 & $\tilde{o}$ & 62 & 42 & & 42 & 62 & \\
\hline 冬 & & $\%$ & 24.0 & 76.0 & & 59.6 & 40.4 & & 40.4 & 59.6 & \\
\hline & Obesity & $\mathrm{N}$ & 15 & 39 & & 28 & 26 & ஓे & 25 & 29 & 5 \\
\hline & & $\%$ & 27.8 & 72.2 & & 51.9 & 48.1 & ஸุ & 46.3 & 53.7 & 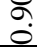 \\
\hline & First & $\mathrm{N}$ & 22 & 39 & & 33 & 28 & & 26 & 35 & \\
\hline & & $\%$ & 36.1 & 63.9 & & 54.1 & 45.9 & & 42.6 & 57.4 & \\
\hline & Second & $\mathrm{N}$ & 22 & 37 & & 33 & 26 & & 30 & 29 & \\
\hline & & $\%$ & 37.3 & 62.7 & & 55.9 & 44.1 & & 50.8 & 49.2 & \\
\hline & Third & $\mathrm{N}$ & 7 & 45 & & 22 & 30 & & 12 & 40 & \\
\hline పై & & $\%$ & 13.5 & 86.5 & & 42.3 & 57.7 & & 23.1 & 76.9 & \\
\hline$\tilde{e}$ & Fourth & $\mathrm{N}$ & 18 & 57 & $\stackrel{\infty}{\curvearrowright}$ & 40 & 35 & & 32 & 43 & \\
\hline$\frac{\tilde{\Xi}}{0}$ & & $\%$ & 24.0 & 76.0 & $\stackrel{0}{0}$ & 53.3 & 46.7 & & 42.7 & 57.3 & \\
\hline 巳ँ & Fifth & $\mathrm{N}$ & 13 & 43 & & 27 & 29 & & 23 & 33 & \\
\hline & & $\%$ & 23.2 & 76.8 & & 48.2 & 51.8 & & 41.1 & 58.9 & \\
\hline & Sixth & $\mathrm{N}$ & 24 & 67 & & 53 & 38 & & 44 & 47 & \\
\hline & & $\%$ & 26.4 & 73.6 & & 58.2 & 41.8 & & 48.4 & 51.6 & \\
\hline & Intern or graduate & $\mathrm{N}$ & 2 & 5 & & 4 & 3 & $\stackrel{\sim}{n}$ & 2 & 5 & 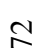 \\
\hline & & $\%$ & 28.6 & 71.4 & & 57.1 & 42.9 & : & 28.6 & 71.4 & . \\
\hline
\end{tabular}

Table 3: Table 2: Comparison of prevalence of prevalence of lower back pain between different colleges

\begin{tabular}{|c|c|c|c|c|c|c|c|c|c|c|c|}
\hline & & & Annu & evalence & & Poin & valence & & & pain & \& \\
\hline & & & No & Yes & $\overline{7}$ & No & Yes & $\overline{7}$ & No & Yes & $\overline{7}$ \\
\hline & Medicine & $\mathrm{N}$ & 28 & 117 & & 75 & 70 & & 62 & 83 & \\
\hline & & $\%$ & 19.3 & 80.7 & & 51.7 & 48.3 & & 42.8 & 57.2 & \\
\hline & Applied Medical Sciences & $\mathrm{N}$ & 10 & 31 & & 21 & 20 & & 11 & 30 & \\
\hline & & $\%$ & 24.4 & 75.6 & & 51.2 & 48.8 & & 26.8 & 73.2 & \\
\hline & Pharmacy & $\mathrm{N}$ & 12 & 15 & & 18 & 9 & & 15 & 12 & \\
\hline & & $\%$ & 44.4 & 55.6 & & 66.7 & 33.3 & & 55.6 & 44.4 & \\
\hline & Dentistry & $\mathrm{N}$ & 2 & 2 & & 3 & 1 & & 4 & 0 & \\
\hline & & $\%$ & 50.0 & 50.0 & & 75.0 & 25.0 & & 100 & 0.0 & \\
\hline & Community Service and & $\mathrm{N}$ & 0 & 4 & & 3 & 1 & & 1 & 3 & \\
\hline & Continuing Education. & $\%$ & 0.0 & 100 & & 75.0 & 25.0 & & 25.0 & 75.0 & \\
\hline & Science & $\mathrm{N}$ & 9 & 12 & & 11 & 10 & & 12 & 9 & \\
\hline & & $\%$ & 42.9 & 57.1 & & 52.4 & 47.6 & & 57.1 & 42.9 & \\
\hline 80 & Engineering & $\mathrm{N}$ & 3 & 15 & & 4 & 14 & & 4 & 14 & \\
\hline$\overline{0}$ & & $\%$ & 16.7 & 83.3 & & 22.2 & 77.8 & & 22.2 & 77.8 & \\
\hline & Administrative and Financial & $\mathrm{N}$ & 4 & 27 & & 13 & 18 & & 7 & 24 & \\
\hline & Sciences & $\%$ & 12.9 & 87.1 & & 41.9 & 58.1 & & 22.6 & 77.4 & \\
\hline & Computing and Information & $\mathrm{N}$ & 11 & 27 & & 23 & 15 & & 13 & 25 & \\
\hline & Technology & $\%$ & 28.9 & 71.1 & & 60.5 & 39.5 & & 34.2 & 65.8 & \\
\hline & Arts & $\mathrm{N}$ & 6 & 9 & & 11 & 4 & & 10 & 5 & \\
\hline & & $\%$ & 40.0 & 60.0 & & 73.3 & 26.7 & & 66.7 & 33.3 & \\
\hline & Design and Applied Arts & $\mathrm{N}$ & 5 & 10 & & 6 & 9 & & 6 & 9 & \\
\hline & & $\%$ & 33.3 & 66.7 & & 40.0 & 60.0 & & 40.0 & 60.0 & \\
\hline & Faculty of Education & $\mathrm{N}$ & 12 & 11 & & 13 & 10 & & 13 & 10 & \\
\hline & & $\%$ & 52.2 & 47.8 & & 56.5 & 43.5 & & 56.5 & 43.5 & \\
\hline & Sharia and Regulations & $\mathrm{N}$ & 6 & 13 & 5 & 11 & 8 & gi & 11 & 8 &  \\
\hline & & $\%$ & 31.6 & 68.4 & 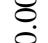 & 57.9 & 42.1 & 4. & 57.9 & 42.1 & ○. \\
\hline
\end{tabular}


Table 4: Mean scores for the domains of RAND 36-Item Health Survey

\begin{tabular}{|l|l|l|l|l|l|l|}
\hline & N of item & Alpha (Internal consistency) & Mean & SD & Minimum & Maximum \\
\hline Physical functioning & 10 & 0.953 & 61.2 & 33.9 & 0.0 & 100 \\
\hline Role limitations due to physical health & 4 & 0.840 & 66.9 & 38.7 & 0.0 & 100 \\
\hline Role limitations due to emotional problems & 3 & 0.840 & 54.6 & 43.4 & 0.0 & 100 \\
\hline Energy/fatigue & 4 & 0.524 & 48.5 & 24.2 & 0.0 & 100 \\
\hline Emotional wellbeing & 5 & 0.641 & 54.3 & 24.3 & 0.0 & 100 \\
\hline Social functioning & 2 & 0.789 & 59.8 & 27.8 & 0.0 & 100 \\
\hline Pain & 2 & 0.687 & 75.1 & 24.1 & 0.0 & 100 \\
\hline General health & 5 & 0.460 & 63.1 & 17.0 & 15.0 & 100 \\
\hline Over change in health & 1 & - & 63.3 & 29.3 & 0.0 & 100 \\
\hline
\end{tabular}

Table 5: Comparison of domain scores between two genders $(n=232)$

\begin{tabular}{|c|c|c|c|c|c|}
\hline & & $\mathbf{N}$ & Mean & SD & $P$ value \\
\hline \multirow[t]{2}{*}{ Physical functioning } & Female & 109 & 64.0 & 28.9 & \multirow[t]{2}{*}{0.853} \\
\hline & Male & 123 & 63.3 & 34.6 & \\
\hline \multirow[t]{2}{*}{ Role limitations due to physical health } & Female & 109 & 58.5 & 39.6 & \multirow[t]{2}{*}{0.515} \\
\hline & Male & 123 & 62.0 & 42.0 & \\
\hline \multirow[t]{2}{*}{ Role limitations due to emotional problems } & Female & 109 & 43.7 & 42.7 & \multirow[t]{2}{*}{0.434} \\
\hline & Male & 123 & 48.2 & 44.6 & \\
\hline \multirow[t]{2}{*}{ Energy/fatigue } & Female & 109 & 38.3 & 23.4 & \multirow[t]{2}{*}{0.004} \\
\hline & Male & 123 & 47.4 & 23.3 & \\
\hline \multirow[t]{2}{*}{ Emotional wellbeing } & Female & 109 & 49.0 & 22.4 & \multirow[t]{2}{*}{0.106} \\
\hline & Male & 123 & 54.1 & 25.6 & \\
\hline \multirow{2}{*}{ Social functioning } & Female & 109 & 55.0 & 28.3 & \multirow[t]{2}{*}{0.445} \\
\hline & Male & 123 & 57.9 & 29.0 & \\
\hline \multirow[t]{2}{*}{ Pain } & Female & 109 & 65.4 & 23.9 & \multirow[t]{2}{*}{0.049} \\
\hline & Male & 123 & 71.8 & 25.3 & \\
\hline \multirow[t]{2}{*}{ General health } & Female & 109 & 58.8 & 17.2 & \multirow[t]{2}{*}{0.046} \\
\hline & Male & 123 & 63.3 & 17.1 & \\
\hline \multirow[t]{2}{*}{ Over all change in health } & Female & 109 & 57.3 & 24.1 & \multirow[t]{2}{*}{0.054} \\
\hline & Male & 123 & 64.4 & 30.7 & \\
\hline
\end{tabular}

Table 6: Comparison of domain scores between different academic years $(n=232)$

\begin{tabular}{|c|c|c|c|c|c|c|c|c|c|c|}
\hline & & $\begin{array}{l}\text { Physical } \\
\text { functioning }\end{array}$ & $\begin{array}{l}\text { Role } \\
\text { limitations } \\
\text { due to } \\
\text { physical } \\
\text { health }\end{array}$ & $\begin{array}{l}\text { Role } \\
\text { limitations } \\
\text { due to } \\
\text { emotional } \\
\text { problems }\end{array}$ & $\begin{array}{l}\text { Energy } \\
\text { /fatigue }\end{array}$ & $\begin{array}{l}\text { Emotional } \\
\text { wellbeing }\end{array}$ & $\begin{array}{l}\text { Social } \\
\text { functioning }\end{array}$ & Pain & $\begin{array}{l}\text { General } \\
\text { health }\end{array}$ & $\begin{array}{l}\text { Overall } \\
\text { health } \\
\text { change }\end{array}$ \\
\hline \multirow[t]{8}{*}{$\begin{array}{l}\text { Mean } \\
\text { (SD) }\end{array}$} & First & $\begin{array}{l}54.5 \\
(32.3)\end{array}$ & $\begin{array}{l}59.4 \\
(40.6)\end{array}$ & $\begin{array}{l}50.8 \\
(43.3)\end{array}$ & $\begin{array}{l}45.2 \\
(24.1)\end{array}$ & $\begin{array}{l}46.2 \\
(22.5)\end{array}$ & $\begin{array}{l}49.4 \\
(29.5)\end{array}$ & $\begin{array}{l}72.6 \\
(27.5)\end{array}$ & $\begin{array}{l}55.9 \\
(16.9)\end{array}$ & $\begin{array}{l}59.4 \\
(29.6)\end{array}$ \\
\hline & Second & $\begin{array}{l}55.2 \\
(33.7)\end{array}$ & $\begin{array}{l}58.1 \\
(40.9)\end{array}$ & $\begin{array}{l}48.6 \\
(44.8)\end{array}$ & $\begin{array}{l}44.7 \\
(26.2)\end{array}$ & $\begin{array}{l}47.9 \\
(20.7)\end{array}$ & $\begin{array}{l}50.4 \\
(25.0)\end{array}$ & $\begin{array}{l}66.7 \\
(27.6)\end{array}$ & $\begin{array}{l}57.3 \\
(19.1)\end{array}$ & $\begin{array}{l}59.3 \\
(31.1)\end{array}$ \\
\hline & Third & $\begin{array}{l}66.0 \\
(31.0)\end{array}$ & $\begin{array}{l}62.0 \\
(40.4)\end{array}$ & $\begin{array}{l}46.8 \\
(43.9)\end{array}$ & $\begin{array}{l}46.8 \\
(26.0)\end{array}$ & $\begin{array}{l}55.5 \\
(26.1)\end{array}$ & $\begin{array}{l}61.8 \\
(26.5)\end{array}$ & $\begin{array}{l}67.1 \\
(26.1)\end{array}$ & $\begin{array}{l}60.7 \\
(16.5)\end{array}$ & $\begin{array}{l}57.2 \\
(34.1)\end{array}$ \\
\hline & Fourth & $\begin{array}{l}54.1 \\
(33.9)\end{array}$ & $\begin{array}{l}63.3 \\
(38.0)\end{array}$ & $\begin{array}{l}52.9 \\
(42.5)\end{array}$ & $\begin{array}{l}54.7 \\
(21.8)\end{array}$ & $\begin{array}{l}57.7 \\
(24.8)\end{array}$ & $\begin{array}{l}62.0 \\
(26.6)\end{array}$ & $\begin{array}{l}80.5 \\
(20.2)\end{array}$ & $\begin{array}{l}64.1 \\
(14.1)\end{array}$ & $\begin{array}{l}67.7 \\
(28.7)\end{array}$ \\
\hline & Fifth & $\begin{array}{l}64.0 \\
(35.7)\end{array}$ & $\begin{array}{l}76.8 \\
(33.3)\end{array}$ & $\begin{array}{l}61.9 \\
(41.4)\end{array}$ & $\begin{array}{l}42.6 \\
(21.8)\end{array}$ & $\begin{array}{l}49.2 \\
(21.8)\end{array}$ & $\begin{array}{l}61.4 \\
(26.0)\end{array}$ & $\begin{array}{l}76.9 \\
(22.0)\end{array}$ & $\begin{array}{l}65.8 \\
(16.8)\end{array}$ & $\begin{array}{l}63.8 \\
(28.2)\end{array}$ \\
\hline & Sixth & $\begin{array}{l}71.4 \\
(32.8)\end{array}$ & $\begin{array}{l}76.9 \\
(36.4)\end{array}$ & $\begin{array}{l}61.2 \\
(43.7)\end{array}$ & $\begin{array}{l}51.6 \\
(24.3)\end{array}$ & $\begin{array}{l}62.2 \\
(24.6)\end{array}$ & $\begin{array}{l}67.6 \\
(28.2)\end{array}$ & $\begin{array}{l}81.8 \\
(18.9)\end{array}$ & $\begin{array}{l}69.3 \\
(15.1)\end{array}$ & $\begin{array}{l}67.9 \\
(25.6)\end{array}$ \\
\hline & $\begin{array}{l}\text { Intern or } \\
\text { graduate }\end{array}$ & $\begin{array}{l}55.0 \\
(40.9)\end{array}$ & $\begin{array}{l}71.4 \\
(36.6)\end{array}$ & $\begin{array}{l}71.4 \\
(40.5)\end{array}$ & $\begin{array}{l}61.4 \\
(21.5)\end{array}$ & $\begin{array}{l}71.4 \\
(23.0)\end{array}$ & $\begin{array}{l}78.6 \\
(18.7)\end{array}$ & $\begin{array}{l}65.4 \\
(26.8)\end{array}$ & $\begin{array}{l}78.6 \\
(16.5)\end{array}$ & $\begin{array}{l}67.9 \\
(27.8)\end{array}$ \\
\hline & & 0.007 & 0.010 & 0.228 & 0.023 & $<0.001$ & $<0.001$ & $<0.001$ & $<0.001$ & 0.207 \\
\hline
\end{tabular}


Table 7: Comparison of domain scores between different colleges $(\mathbf{n}=\mathbf{2 3 2})$

\begin{tabular}{|c|c|c|c|c|c|c|c|c|c|c|}
\hline & & $\begin{array}{l}\text { Physical } \\
\text { function- } \\
\text { ing }\end{array}$ & $\begin{array}{l}\text { Role } \\
\text { limitations } \\
\text { due to } \\
\text { physical } \\
\text { health }\end{array}$ & $\begin{array}{l}\text { Role } \\
\text { limitations } \\
\text { due to } \\
\text { emotional } \\
\text { problems }\end{array}$ & $\begin{array}{l}\text { Energy/ } \\
\text { fatigue }\end{array}$ & $\begin{array}{l}\text { Emotional } \\
\text { wellbeing }\end{array}$ & $\begin{array}{l}\text { Social } \\
\text { function- } \\
\text { ing }\end{array}$ & Pain & $\begin{array}{l}\text { General } \\
\text { health }\end{array}$ & $\begin{array}{l}\text { Overall } \\
\text { health } \\
\text { change }\end{array}$ \\
\hline \multirow[t]{13}{*}{$\begin{array}{l}\text { Mean } \\
(\mathrm{sd})\end{array}$} & Medicine & $\begin{array}{l}66.1 \\
(35.5)\end{array}$ & $\begin{array}{l}68.6 \\
(40.4)\end{array}$ & $\begin{array}{l}56.1 \\
(43.5)\end{array}$ & $\begin{array}{l}45.7 \\
(23.9)\end{array}$ & $\begin{array}{l}52.4 \\
(24.5)\end{array}$ & $\begin{array}{l}61.6 \\
(28.5)\end{array}$ & $\begin{array}{l}79.8 \\
(20.9)\end{array}$ & $\begin{array}{l}67 . \\
(17.1)\end{array}$ & $\begin{array}{l}63.4 \\
(28.7)\end{array}$ \\
\hline & $\begin{array}{l}\text { Applied Medical } \\
\text { Sciences }\end{array}$ & $\begin{array}{l}58.2 \\
(32.9)\end{array}$ & $\begin{array}{l}61.6 \\
(39.6)\end{array}$ & $\begin{array}{l}43.1 \\
(44.2)\end{array}$ & $\begin{array}{l}39.9 \\
(29.8)\end{array}$ & $\begin{array}{l}46.8 \\
(29.4)\end{array}$ & $\begin{array}{l}48.5 \\
(29.7)\end{array}$ & $\begin{array}{l}70.6 \\
(24.7)\end{array}$ & $\begin{array}{l}56.6 \\
(18.4)\end{array}$ & $\begin{array}{l}59.8 \\
(29.0)\end{array}$ \\
\hline & Pharmacy & $\begin{array}{l}40.2 \\
(32.9)\end{array}$ & $\begin{array}{l}66.7 \\
(39.2)\end{array}$ & $\begin{array}{l}71.6 \\
(37.8) \\
\end{array}$ & $\begin{array}{l}55.9 \\
(18.1) \\
\end{array}$ & $\begin{array}{l}53.8 \\
(19.8) \\
\end{array}$ & $\begin{array}{l}50.0 \\
(21.7) \\
\end{array}$ & $\begin{array}{l}74.4 \\
(21.5) \\
\end{array}$ & $\begin{array}{l}59.3 \\
(15.9) \\
\end{array}$ & $\begin{array}{l}67.6 \\
(33.1) \\
\end{array}$ \\
\hline & Dentistry & $\begin{array}{l}30.0 \\
(22.7)\end{array}$ & $\begin{array}{l}93.8 \\
(12.5)\end{array}$ & $\begin{array}{l}91.7 \\
(16.7)\end{array}$ & $\begin{array}{l}53.8 \\
(7.5)\end{array}$ & $\begin{array}{l}49.0 \\
(6.8)\end{array}$ & $\begin{array}{l}46.9 \\
(35.9)\end{array}$ & $\begin{array}{l}91.3 \\
(11.8)\end{array}$ & $\begin{array}{l}57.5 \\
(26.0)\end{array}$ & $\begin{array}{l}93.8 \\
(12.5)\end{array}$ \\
\hline & $\begin{array}{l}\text { Community Service } \\
\text { and Continuing } \\
\text { Education. }\end{array}$ & $\begin{array}{l}45.0 \\
(34.9)\end{array}$ & $\begin{array}{l}68.8 \\
(47.3)\end{array}$ & $\begin{array}{l}58.3 \\
(50.0)\end{array}$ & $\begin{array}{l}56.3 \\
(11.8)\end{array}$ & $\begin{array}{l}78.0 \\
(27.2)\end{array}$ & $\begin{array}{l}68.8 \\
(37.5)\end{array}$ & $\begin{array}{l}64.4 \\
(47.4)\end{array}$ & $\begin{array}{l}68.8 \\
(30.7)\end{array}$ & $\begin{array}{l}50 \\
(40.8)\end{array}$ \\
\hline & Science & $\begin{array}{l}51.7 \\
(37.1)\end{array}$ & $\begin{array}{l}53.6 \\
(44.9)\end{array}$ & $\begin{array}{l}42.9 \\
(46.1)\end{array}$ & $\begin{array}{l}57.4 \\
(15.3)\end{array}$ & $\begin{array}{l}62.5 \\
(18.6)\end{array}$ & $\begin{array}{l}64.3 \\
(23.1)\end{array}$ & $\begin{array}{l}73.5 \\
(34.4)\end{array}$ & $\begin{array}{l}61.2 \\
(21.0)\end{array}$ & $\begin{array}{l}59.5 \\
(32.1)\end{array}$ \\
\hline & Engineering & $\begin{array}{l}70.3 \\
(30.1)\end{array}$ & $\begin{array}{l}52.8 \\
(40.1)\end{array}$ & $\begin{array}{l}51.9 \\
(44.6)\end{array}$ & $\begin{array}{l}45.6 \\
(26.8)\end{array}$ & $\begin{array}{l}56.2 \\
(22.6)\end{array}$ & $\begin{array}{l}56.9 \\
(25.4)\end{array}$ & $\begin{array}{l}69.9 \\
(23.1)\end{array}$ & $\begin{array}{l}64.4 \\
(15.5)\end{array}$ & $\begin{array}{l}61.1 \\
(28.7)\end{array}$ \\
\hline & $\begin{array}{l}\text { Administrative and } \\
\text { Financial Sciences }\end{array}$ & $\begin{array}{l}64.7 \\
(27.2)\end{array}$ & $\begin{array}{l}70.2 \\
(31.9)\end{array}$ & $\begin{array}{l}50.5 \\
(45.4)\end{array}$ & $\begin{array}{l}47.9 \\
(25.5)\end{array}$ & $\begin{array}{l}59.0 \\
(23.4)\end{array}$ & $\begin{array}{l}66.5 \\
(27.5)\end{array}$ & $\begin{array}{l}71.9 \\
(18.3)\end{array}$ & $\begin{array}{l}64.2 \\
(13.5)\end{array}$ & $\begin{array}{l}60.5 \\
(24.8)\end{array}$ \\
\hline & $\begin{array}{l}\text { Computing and } \\
\text { Information } \\
\text { Technology }\end{array}$ & $\begin{array}{l}69.6 \\
(32.2)\end{array}$ & $\begin{array}{l}68.4 \\
(36.6)\end{array}$ & $\begin{array}{l}56.1 \\
(44.6)\end{array}$ & $\begin{array}{l}50.3 \\
(28.6)\end{array}$ & $\begin{array}{l}56.9 \\
(22.0)\end{array}$ & $\begin{array}{l}63.2 \\
(30.6)\end{array}$ & $\begin{array}{l}73.4 \\
(25.4)\end{array}$ & $\begin{array}{l}60.4 \\
(17.1)\end{array}$ & $\begin{array}{l}59.2 \\
(30.4)\end{array}$ \\
\hline & Arts & $\begin{array}{l}73.0 \\
(34.7) \\
\end{array}$ & $\begin{array}{l}88.3 \\
(20.8) \\
\end{array}$ & $\begin{array}{l}77.8 \\
(41.1) \\
\end{array}$ & $\begin{array}{l}68.7 \\
(22.8) \\
\end{array}$ & $\begin{array}{l}72.3 \\
(27.6) \\
\end{array}$ & $\begin{array}{l}74.2 \\
(24.3) \\
\end{array}$ & $\begin{array}{l}76.7 \\
(24.9) \\
\end{array}$ & $\begin{array}{l}62.3 \\
(16.9) \\
\end{array}$ & $\begin{array}{l}68.3 \\
(34.7) \\
\end{array}$ \\
\hline & $\begin{array}{l}\text { Design and Applied } \\
\text { Arts }\end{array}$ & $\begin{array}{l}53.7 \\
(24.3)\end{array}$ & $\begin{array}{l}45.0 \\
(44.5)\end{array}$ & $\begin{array}{l}33.3 \\
(35.6)\end{array}$ & $\begin{array}{l}34.0 \\
(18.8)\end{array}$ & $\begin{array}{l}30.1 \\
(12.8)\end{array}$ & $\begin{array}{l}50.0 \\
(25.0)\end{array}$ & $\begin{array}{l}52.2 \\
(32.7)\end{array}$ & $\begin{array}{l}54.7 \\
(14.7)\end{array}$ & $\begin{array}{l}63.3 \\
(32.6)\end{array}$ \\
\hline & $\begin{array}{l}\text { Faculty of } \\
\text { Education }\end{array}$ & $\begin{array}{l}48.7 \\
(35.5)\end{array}$ & $\begin{array}{l}73.9 \\
(35.7)\end{array}$ & $\begin{array}{l}60.9 \\
(41.0)\end{array}$ & $\begin{array}{l}54.1 \\
(17.8)\end{array}$ & $\begin{array}{l}52.5 \\
(21.2)\end{array}$ & $\begin{array}{l}59.2 \\
(22.7)\end{array}$ & $\begin{array}{l}77.9 \\
(24.6)\end{array}$ & $\begin{array}{l}63.9 \\
(13.6)\end{array}$ & $\begin{array}{l}66.3 \\
(28.8)\end{array}$ \\
\hline & $\begin{array}{l}\text { Sharia and } \\
\text { Regulations }\end{array}$ & $\begin{array}{l}61.1 \\
(25.2)\end{array}$ & $\begin{array}{l}71.1 \\
(30.3)\end{array}$ & $\begin{array}{l}45.6 \\
(37.2)\end{array}$ & $\begin{array}{l}53.9 \\
(14.3)\end{array}$ & $\begin{array}{l}65.1 \\
(18.6)\end{array}$ & $\begin{array}{l}62.5 \\
(25.7)\end{array}$ & $\begin{array}{l}77.2 \\
(20.3)\end{array}$ & $\begin{array}{l}63.2 \\
(10.6)\end{array}$ & $\begin{array}{l}72.4 \\
(24.9)\end{array}$ \\
\hline \multicolumn{2}{|c|}{$\mathrm{P}$ value } & 0.003 & 0.097 & 0.043 & 0.001 & $<0.001$ & 0.043 & 0.012 & 0.058 & 0.127 \\
\hline
\end{tabular}

Table 8: Comparison of domain scores according to BMI $(n=232)$

\begin{tabular}{|c|c|c|c|c|c|}
\hline & & $\mathrm{N}$ & Mean & SD & \\
\hline \multirow[t]{4}{*}{ Physical functioning } & Underweight & 39 & 57.6 & 33.0 & \multirow[t]{4}{*}{0.115} \\
\hline & Normal weight & 101 & 65.1 & 32.8 & \\
\hline & Overweight & 62 & 69.2 & 30.3 & \\
\hline & Obesity & 29 & 54.3 & 30.1 & \\
\hline \multirow{4}{*}{$\begin{array}{l}\text { Role limitations due to physical } \\
\text { health }\end{array}$} & Underweight & 39 & 35.9 & 42.8 & \multirow[t]{4}{*}{$<0.001$} \\
\hline & Normal weight & 101 & 68.1 & 37.3 & \\
\hline & Overweight & 62 & 64.1 & 39.6 & \\
\hline & Obesity & 29 & 57.8 & 42.3 & \\
\hline \multirow{4}{*}{$\begin{array}{l}\text { Role limitations due to emotional } \\
\text { problems }\end{array}$} & Underweight & 39 & 27.4 & 34.1 & \multirow[t]{4}{*}{0.014} \\
\hline & Normal weight & 101 & 46.5 & 44.5 & \\
\hline & Overweight & 62 & 50.0 & 45.1 & \\
\hline & Obesity & 29 & 59.8 & 43.1 & \\
\hline \multirow[t]{4}{*}{ Energy/fatigue } & Underweight & 39 & 37.7 & 20.8 & \multirow[t]{4}{*}{0.153} \\
\hline & Normal weight & 101 & 41.5 & 24.0 & \\
\hline & Overweight & 62 & 45.7 & 25.0 & \\
\hline & Obesity & 29 & 49.3 & 22.1 & \\
\hline \multirow[t]{4}{*}{ Emotional wellbeing } & Underweight & 39 & 41.4 & 20.6 & \multirow[t]{4}{*}{$<0.001$} \\
\hline & Normal weight & 101 & 49.0 & 22.5 & \\
\hline & Overweight & 62 & 55.3 & 24.4 & \\
\hline & Obesity & 29 & 65.9 & 26.8 & \\
\hline \multirow[t]{3}{*}{ Social functioning } & Underweight & 39 & 47.4 & 26.2 & \multirow[t]{3}{*}{0.148} \\
\hline & Normal weight & 101 & 57.2 & 30.8 & \\
\hline & Overweight & 62 & 58.5 & 26.2 & \\
\hline
\end{tabular}




\begin{tabular}{|c|c|c|c|c|c|}
\hline & Obesity & 29 & 62.1 & 27.6 & \\
\hline \multirow[t]{4}{*}{ Pain } & Underweight & 39 & 66.7 & 26.7 & \multirow[t]{4}{*}{0.915} \\
\hline & Normal weight & 101 & 69.3 & 22.1 & \\
\hline & Overweight & 62 & 69.9 & 25.2 & \\
\hline & Obesity & 29 & 67.4 & 31.4 & \\
\hline \multirow[t]{4}{*}{ General health } & Underweight & 39 & 55.8 & 18.8 & \multirow[t]{4}{*}{0.043} \\
\hline & Normal weight & 101 & 61.6 & 16.6 & \\
\hline & Overweight & 62 & 65.1 & 18.0 & \\
\hline & Obesity & 29 & 57.9 & 14.0 & \\
\hline \multirow[t]{4}{*}{ Overall health change } & Underweight & 39 & 53.8 & 32.7 & \multirow[t]{4}{*}{0.303} \\
\hline & Normal weight & 101 & 62.9 & 24.7 & \\
\hline & Overweight & 62 & 63.7 & 28.2 & \\
\hline & Obesity & 29 & 59.5 & 31.6 & \\
\hline
\end{tabular}

Table 9: Comparison of total scores of Quality of life according to different prevalence $(n=401)$

\begin{tabular}{|l|l|l|l|l|l|}
\hline Prevalence & Lower back pain & N & Mean & Std. Deviation & P value \\
\hline \multirow{2}{*}{ Life time prevalence } & Yes & 232 & 512.5517 & 172.52550 & $<0.001$ \\
\cline { 2 - 6 } & No & 169 & 593.7130 & 149.91738 & \\
\hline \multirow{2}{*}{ Annual prevalence } & Yes & 293 & 516.5722 & 169.16544 & $<0.001$ \\
\cline { 2 - 7 } & No & 108 & 628.6466 & 134.93793 & 164.35878 \\
\hline \multirow{2}{*}{ Point prevalence } & Yes & 189 & 475.8501 & 144.72767 & $<0.001$ \\
\cline { 2 - 7 } & No & 212 & 609.9709 & 12 & \\
\hline
\end{tabular}

Covariates: Annual prevalence, Gender, academic year, college and BMI

Table 10: Regression model for impact of LBP on QOL $(n=401)$

\begin{tabular}{|c|c|c|c|c|c|}
\hline \multicolumn{6}{|c|}{ Dependent Variable: Total Quality of life scores } \\
\hline Source & Type III Sum of Squares & $\mathrm{df}$ & Mean Square & $\mathrm{F}$ & $\mathrm{P}$ value \\
\hline Corrected Model & $2098556.055^{\mathrm{a}}$ & 5 & 419711.211 & 18.026 & .000 \\
\hline Intercept & 3321845.378 & 1 & 3321845.378 & 142.668 & .000 \\
\hline Gender & 6801.890 & 1 & 6801.890 & .292 & .589 \\
\hline Academic year & 843582.792 & 1 & 843582.792 & 36.231 & .000 \\
\hline College & 7148.426 & 1 & 7148.426 & .307 & .580 \\
\hline BMI & 139040.487 & 1 & 139040.487 & 5.972 & .015 \\
\hline Annual prevalence & 1068965.854 & 1 & 1068965.854 & 45.910 & .000 \\
\hline Error & 9197070.433 & 395 & 23283.723 & & \\
\hline Total & 131171794.194 & 401 & & & \\
\hline Corrected Total & 11295626.488 & 400 & & & \\
\hline
\end{tabular}

\section{Discussion}

Lower back pain is one of the most common reasons for absence from attending work and college after upper respiratory tract infections ${ }^{[23]}$. This study's findings showed that lower back pain (LBP) was very much higher and had significantly impacted the quality of life (QOL) of students. The incidence of LBP creates an economic burden due to reduced efficiency and productivity at the workplace ${ }^{[24]}$. Even though there is no clear cause for LBP, some of the factors that could trigger this kind of non-specific pain include tense muscles, reduced movement, and weak core muscles, prolonged sitting in one position, strenuous physical activities, psychological stress at the workplace or college or family, changes in perception of pain due to changes in the central nervous system, genetic predisposition ${ }^{[25-27]}$. A study done in Saudi Arabia among male University students reported that the prevalence of LBP was $30 \%{ }^{[28]}$, which is comparatively lesser than the prevalence found in our study for male students. Among university students, one of the common causes of the LBP could be attributed to emotional stress ${ }^{[29]}$, and it is evident that many psychosocial factors such as poor social support, high job demands, and poor job control play an important role in the etiology of musculoskeletal pain ${ }^{[30]}$. Another recent study conducted among Medical Students of Taif
University reported an LBP prevalence of $33.3 \%{ }^{[20]}$, which was lesser than the prevalence of LBP among medical students in our study $(57.2 \%)$.

A study done by Alshagga et al. among undergraduate medical students reported that long working hours was one of the contributory factors for LBP ${ }^{[31]}$. We have the opinion that the higher prevalence of the LBP could not be attributed to long hours of sitting during the lectures as most of the lectures were conducted online due to COVID-19 ${ }^{[32]}$. Obesity is considered a risk factor for LBP development due to the biomechanical and inflammatory effects of increased body weight on the spine ${ }^{[33]}$. In our study, we didn't find any significant effect of higher BMI on LBP prevalence. It was reported that LBP has a major impact on various physical and psychosocial health domains, and it is the major reason for limited activity in adults less than 45 years old ${ }^{[34,35]}$. Although many studies have investigated the impact of LBP on the QOL, there is a lack of studies in Saudi Arabia that assessed and compared the impact of LBP on QOL of different specialties of university students. This study's findings showed that LBP had a significant impact on various domains, including physical, Emotional, and Social health. A study done among physiotherapy students in Zimbabwe reported a lifetime prevalence of LBP to be $56.7 \%$ and showed a significant association in lowering physical 
disability ${ }^{[36]}$. University students are at higher risk of developing LBP due to improper posture, reduced physical activity, sedentary lifestyle, and physiological stress ${ }^{[37-39]}$. Moroder et al. had reported that medical students are 2.5 times less physically active than nonscience students, which is a major reason for the higher incidence of LBP among medical students ${ }^{[40]}$. It is also reported that female students have an increased risk of developing LBP compared to males. This could be due to reduced physical and sports activities, high sedentary activities, and insufficient quantity and quality of sleep compared to male students ${ }^{[41-43]}$. These findings are consistent with our study findings where female students showed a higher annual prevalence of LBP even though there were no significant differences in its impact on QOL. Health-related quality of life is considered a crucial outcome when investigating LBP. Our study's findings show that chronic LBP negatively impacted the Health-related quality of life of university students. The incidence of LBP could reduce the future productivity and efficiency at the workplace when these students begin to work in their respective fields. Further research is warranted to identify additional contributory factors that could help develop a specific intervention to reduce the incidence of LBP among University students and minimize the burden on both students and parents. One of the limitations of this study is its cross-sectional nature, which may have prevented us from making a conclusion regarding the causality. There is another possibility for bias that may have occurred due to underreporting of LBP among the students. All of the above limitations should be considered before generalizing our study findings.

\section{Conclusion}

This cross-sectional study showed that lower back pain had negatively impacted the health-related quality of life of Taif university students. Female students had a higher prevalence of LBP than males, but there were no significant differences in the impact on quality of life. In students who showed an annual prevalence of LBP, lower academic year and increased BMI were found to affect the quality of life significantly. There is a need to increase the awareness among University students regarding various risk factors for LBP and their relationship with Healthrelated quality of life.

\section{Ethics approval}

Institutional research ethics board approval was acquired before conducting any study-related procedures. A statement was included at the beginning of the questionnaire clarifying that the participation in this study is voluntary and that collected data will be anonymous and will only be used for this study.

\section{Conflicts of Interest}

The authors have no conflicts of interest to declare.

\section{Source of Funding}

This study did not receive any specific grant from funding agencies in the public, commercial, or not-for-profit sectors.

\section{References}

[1] Balagué F, Mannion AF, Pellisé F, Cedraschi C. Nonspecific low back pain. Lancet (London, England). 2012;379(9814):482-91.

[2] Marty M, Rozenberg S, Duplan B, Thomas P, Duquesnoy B, Allaert F, et al. Quality of sleep in patients with chronic low back pain: a case-control study. European Spine Journal. 2008;17(6):839-44.

[3] Mutubuki EN, Beljon Y, Maas ET, Huygen F, Ostelo R, van Tulder MW, et al. The longitudinal relationships between pain severity and disability versus health-related quality of life and costs among chronic low back pain patients. Quality of life research : an international journal of quality of life aspects of treatment, care and rehabilitation. 2020;29(1):275-87.

[4] Geurts JW, Willems PC, Kallewaard JW, van Kleef M, Dirksen C. The Impact of Chronic Discogenic Low Back Pain: Costs and Patients' Burden. Pain research \& management. 2018;2018:4696180.

[5] Yang H, Haldeman S, Lu ML, Baker D. Low Back Pain Prevalence and Related Workplace Psychosocial Risk Factors: A Study Using Data From the 2010 National Health Interview Survey. Journal of manipulative and physiological therapeutics. 2016;39(7):459-72.

[6] Dutmer AL, Schiphorst Preuper HR, Soer R, Brouwer S, Bültmann U, Dijkstra PU, et al. Personal and Societal Impact of Low Back Pain: TheGroningen Spine Cohort. Spine. 2019;44(24):E1443-e51.

[7] Handrakis JP, Friel K, Hoeffner F, Akinkunle O, Genova $\mathrm{V}$, Isakov $\mathrm{E}$, et al. Key characteristics of low back pain and disability in college-aged adults: a pilot study. Archives of physical medicine and rehabilitation. 2012;93(7):1217-24.

[8] Ekman M, Jönhagen S, Hunsche E, Jönsson L. Burden of illness of chronic low back pain in Sweden: a crosssectional, retrospective study in primary care setting. Spine. 2005;30(15):1777-85.

[9] Tagliaferri SD, MillerCT, Owen PJ, Mitchell UH, Brisby H, Fitzgibbon B, et al. Domains of Chronic Low Back Pain and Assessing Treatment Effectiveness: A Clinical Perspective. Pain practice : the official journal of World Institute of Pain. 2020;20(2):211-25.

[10] Hoy D, BrooksP, Blyth F, Buchbinder R. The Epidemiology of low back pain. Best practice \& research Clinical rheumatology. 2010;24(6):769-81.

[11] Manchikanti L, Singh V, Datta S, Cohen SP, Hirsch JA. Comprehensive review of epidemiology, scope, and impact of spinal pain. Pain physician. 2009;12(4):E3570.

[12] Hirano K, Imagama S, Hasegawa Y, Ito Z, Muramoto A, Ishiguro N. Impact of low back pain, knee pain, and timed up-and-go test on quality of life in communityliving people. Journal of Orthopaedic Science. 2014;19(1):164-71.

[13] Zhang Y, Deng G, Zhang Z, Zhou Q, Gao X, Di L, et al. A cross sectional study between the prevalence of chronic pain and academic pressure in adolescents in China (Shanghai). BMC musculoskeletal disorders. 2015;16:219.

[14] Kanchanomai S, Janwantanakul P, Pensri P, Jiamjarasrangsi W. A prospective study of incidence and risk factors for the onset and persistence of low back pain in Thai university students. Asia-Pacific journal of public health. 2015;27(2):Np106-15. 
[15] Kędra A, Kolwicz-Gańko A, Sitarski D, Ewertowska P, Czaprowski D. Low Back Pain and Everyday Functioning of Students. Ortopedia, traumatologia, rehabilitacja. 2016;18(1):31-9.

[16] Algarni AD, Al-Saran Y, Al-Moawi A, Bin Dous A, AlAhaideb A, Kachanathu SJ. The Prevalence of and Factors Associated with Neck, Shoulder, and Low-Back Pains among Medical Students at University Hospitals in Central Saudi Arabia. Pain research and treatment. 2017;2017:1235706.

[17] Kato K, Sekiguchi M, Nikaido T, Otoshi KI, Matsuo Y, Igari T, et al. Psychosocial Stress After a Disaster and Low Back Pain-Related Interference With Daily Living Among College Students: A Cohort Study in Fukushima. Spine. 2017;42(16):1255-60.

[18] AlShayhan FA, Saadeddin M. Prevalence of low back pain among health sciencesstudents. European journal of orthopaedic surgery \& traumatology: orthopedie traumatologie. 2018;28(2):165-70.

[19] Amelot A, Mathon B, Haddad R, Renault MC, Duguet A, Steichen O. Low Back Pain Among Medical Students: A Burden and an Impact to Consider! Spine. 2019;44(19):1390-5.

[20] Alturkistani LH, Hendi OM, Bajaber AS, Alhamoud MA, Althobaiti SS, Alharthi TA, et al. Prevalence of Lower Back Pain and its Relation to Stress Among Medical Students in Taif University, Saudi Arabia. International journal of preventive medicine. 2020;11:35.

[21] Crawford JO. The Nordic musculoskeletal questionnaire. Occupational medicine. 2007;57(4):300-1.

[22] Coons SJ, Alabdulmohsin SA, Draugalis JR, Hays RD. Reliability of an Arabic version of the RAND-36 Health Survey andits equivalence to the US-English version. Medical care. 1998;36(3):428-32.

[23] Chen YL. Effectiveness of a new back belt in the maintenance of lumbar lordosis while sitting: a pilot study. International journal of industrial ergonomics. 2003 Oct 1;32(4):299-303.

[24] Mokhtarinia HR, Hosseini A, Maleki-Ghahfarokhi A, Gabel CP, Zohrabi M. Cross-cultural adaptation, validity, and reliability of the Persian version of the spine functional index. Health Qual Life Outcomes. 2018;16(1):95.

[25] Buchbinder R, van Tulder M, Öberg B, Costa LM, Woolf A, Schoene M, Croft P; Lancet Low Back Pain Series Working Group. Low back pain: a call for action. Lancet. 2018 Jun 9;391(10137):2384-2388.

[26] Brinjikji W, Luetmer PH, Comstock B, Bresnahan BW, Chen LE, Deyo RA, Halabi S, Turner JA, Avins AL, James K, Wald JT, Kallmes DF, Jarvik JG. Systematic literature review of imaging features of spinal degeneration in asymptomatic populations. AJNR Am J Neuroradiol. 2015 Apr;36(4):811-6.

[27] da Silva T, Mills K, Brown BT, Herbert RD, Maher CG, Hancock MJ. Risk of Recurrence of Low Back Pain: A Systematic Review. J Orthop Sports Phys Ther. 2017 May;47(5):305-313.

[28] Issa LF, Seleem NA, Bakheit AM, Baky AA, Alotaibi AF. Low back pain among undergraduate student at Taif University-Saudi Arabia. Int J Public Health Epidemiol. 2016;5(6):275-84.
[29] Rahman AA, Al Hashim BN, Al Hiji NK, Al-Abbad Z. Stress among medical Saudi students at college of medicine, King Faisal University. J Prev Med Hyg. 2013;54:195-9.

[30] Sohail N. Stress and academic performance among medical students. J Coll Physicians Surg Pak. 2013;23:67-71.

[31] Alshagga MA, Nimer AR, Yan LP, Ibrahim IA, AlGhamdi SS, Radman Al-Dubai SA. Prevalence and factors associated with neck, shoulder and low back pains among medical students in a Malaysian Medical College. BMC Res Notes. 2013;6:244.

[32] Hendi OM, Abdulaziz AA, Althaqafi AM, Hindi AM, Khan SA, Atalla AA. Prevalence of musculoskeletal disorders and its correlation to physical activity among health specialty students. Int J Prev Med. 2019;10:48.

[33] Chou L, Brady SRE, Urquhart DM, et al. The Association Between Obesity and Low Back Pain and Disability Is Affected by Mood Disorders: A PopulationBased, Cross-Sectional Study of Men. Medicine (Baltimore). 2016;95(15):e3367.

[34] Waddell G. The Back Pain Revolution. Churchill Livingston: Edinburgh, London, New York. Oxford,Philadelphia, St. Louis, Sydney, Toronto; 2004.

[35] Andersson GB. Epidemiological features of chronic lowback pain. Lancet. 1999 Aug 14;354(9178):581-5.

[36] Chiwaridzo M, Chamarime KJ, Dambi JM. The burden of low back pain among undergraduate physiotherapy students at the University of Zimbabwe: a cross-sectional study. BMC Res Notes. 2018;11(1):697

[37] Holth HS, Werpen HK, Zwart JA, Hagen K. Physical inactivity is associated with chronic musculoskeletal complaints 11 years later: results from the NordTrøndelag Health Study. BMC Musculoskelet Disord. 2008 Dec 1;9:159.

[38] Zvonar M, Kasović M, Štefan L. Physical Fitness and the Level of Pain Intensity in Adolescents: A School-based Study. Int J Environ Res Public Health. 2019 Jul 6;16(13):2410.

[39] Swain MS, Henschke N, Kamper SJ, Gobina I, OttováJordan V, Maher CG. An international survey of pain in adolescents. BMC Public Health. 2014 May 13;14:447.

[40] Moroder P, Runer A, Resch H, Tauber M. Low back pain among medical students. Acta Orthop Belg. 2011 Feb;77(1):88-92.

[41] Dighriri YH, Akkur MA, Alharbi SA, Madkhali NA, Matabi KI, Mahfouz MS. Prevalence and associated factors of neck, shoulder, and low-back pains among medical students at Jazan University, Saudi Arabia: A cross-sectional study. J Family Med Prim Care. 2019;8(12):3826-3831.

[42] Angelone AM, Mattei A, Sbarbati M, Di Orio F. Prevalence and correlates for self-reported sleep problems among nursing students. J Prev Med Hyg. 2011;52:201-8.

[43] Kompal R, Kashif M, Riaz U, Dastgir A, Irum H, Manzoor N. Prevalence of low back, neck and shoulder pain and associated risk factors among senior semester female students of the university of Faisalabad. Int $\mathbf{J}$ Rehabil Sci. 2017;5:21-7. 